\title{
Análisis de artículos de prensa digital para valorar su uso como recurso para la enseñanza de la Naturaleza de la Ciencia en Educación Secundaria
}

\author{
Rocío Jurado Palomares y José Miguel Vílchez González \\ Departamento de Didáctica de las Ciencias Experimentales. Facultad de Ciencias de la \\ Educación. Universidad de Granada. España
}

[Recibido el 16 de julio de 2020, aceptado el 4 de noviembre de 2020]

\begin{abstract}
La enseñanza de la Naturaleza de la Ciencia (NdC) resulta fundamental para el desarrollo de la alfabetización científica. Con el objetivo de impulsar su introducción en el currículo de ciencias, se propone el uso de noticias científicas de la prensa digital, como un recurso sencillo y accesible. La elección de noticias para su uso en el aula requiere un análisis previo, con el fin de visualizar su interés didáctico, y es a ello a lo que se dedica este artículo. Se muestran los resultados de un análisis realizado con 53 noticias relacionadas con el ámbito de las ciencias, publicadas en tres de los diarios digitales más populares de la prensa española. Se concluye que la colección de noticias tiene un contenido amplio y diverso, haciendo alusiones a todos los aspectos de la $\mathrm{NdC}$, por lo que su empleo es totalmente recomendado. Queda abierta una línea de investigación en relación a su uso en el aula y la evaluación de su eficacia.
\end{abstract}

Palabras clave: naturaleza de la ciencia, prensa online, educación.

\section{Analysis of online newspapers to assess their usefulness as a teaching resource for Nature of Science in secondary schools}

Nature of Science (NOS) education is essential for the development of scientific literacy. Scientific articles in online newspapers are a simple and accessible resource for the promotion of NOS within the science curriculum. This article examines the selection of articles based on their educational value and relevance to the curriculum. The study consisted of an analysis of 53 articles related to the field of science from three of the most popular newspapers in the Spanish press. The results showed the articles to be highly suitable as an educational resource owing to the diverse array of content featured on different aspects of NOS. Their use and effectiveness in the classroom remain to be examined in future research.

Keywords: Nature of Science, online newspapers, education.

Para citar el artículo. Jurado Palomares, R. y Vílchez González, J. M. (201x). Análisis de artículos de prensa digital para valorar su uso como recurso para la enseñanza de la Naturaleza de la Ciencia en Educación Secundaria. Ápice. Revista de Educación Científica, 5(1), 53-71. DOI: https:// doi.org/10.17979/arec.2021.5.1.6838

Contacto.rociojp@ugr.es; jmvilchez@ugr.es 


\section{Introducción}

La ciencia surge en la sociedad, por lo que se reconoce como una actividad humana y por ello se ve afectada por el contexto histórico, socioeconómico y por las ideologías imperantes (Vázquez et al., 2007; Vázquez y Manassero, 2012).

Resulta evidente que la sociedad actual se encuentra cada vez más influenciada por la ciencia y la tecnología, lo que hace necesaria una formación de la ciudadanía en temas relacionados con las disciplinas científico-tecnológicas. Por tanto, cabe destacar el papel esencial de la enseñanza de las ciencias en la educación, que ha de derivar en lo que se viene llamando la alfabetización científica.

La alfabetización científica es un lema que actualmente orienta la enseñanza de las ciencias a través de dos componentes básicos: enseñanza de la ciencia (contenidos de conocimientos y procesos) y enseñanza sobre la ciencia (contenidos sobre qué es la ciencia) (García-Carmona et al., 2011). Es en este último punto en el que toma su importancia la Naturaleza de la Ciencia (NdC). La NdC es un componente esencial de la alfabetización científica que implica, entre otras cuestiones, entender los procesos mediante los que se genera y evoluciona el conocimiento científico, apreciar el poder explicativo y predictivo de las teorías científicas, la evolución de éstas y el impacto de sus avances en la sociedad (Romero y Vázquez, 2012).

Por otro lado, la alfabetización científica también requiere la comprensión de la NdC para permitir a los individuos posicionarse frente a temáticas socio-científicas de interés de manera informada, participar activamente en las decisiones tecno-científicas, y que además dichas decisiones sean justificadas y responsables (Acevedo et al., 2005).

Muchos expertos en Didáctica de las Ciencias han apoyado la inclusión de la NdC en el currículo de ciencias para conseguir así dicha participación ciudadana (Driver et al., 1996; McComas et al., 1998; Spector et al., 1998). Aunque tradicionalmente estos currículos se han centrado mayoritariamente en los contenidos conceptuales, existe cada vez un mayor consenso acerca de que un objetivo prioritario de la educación científica es que los estudiantes de Educación Secundaria lleguen a adquirir una mejor comprensión de la NdC (Matthews, 1998; McComas et al., 1998; Ziman, 2000).

Sin embargo, la NdC aún no se ha integrado de un modo efectivo en los procesos de enseñanza-aprendizaje de las ciencias (Acevedo-Díaz y García-Carmona, 2016; AcevedoDíaz et al., 2017; Lederman, 2006). Por otro lado, la literatura especializada también ha constatado que los ciudadanos, los estudiantes e incluso los docentes, poseen visiones simplistas o deformadas sobre la ciencia (Campanario et al., 2001; Fernández et al., 2002; Lederman, 2007; Moreno y Calvo, 2019; Vílchez et al., 2015).

Debido a todo lo anterior, es necesario elaborar y llevar a cabo diferentes propuestas educativas que involucren de manera activa al alumnado de Educación Secundaria, en las que el docente adopte un rol de motivador y guía, implicando a los estudiantes en experiencias que mejoren su comprensión sobre la NdC.

En este sentido, la introducción de noticias científicas en el aula es de gran importancia para que el alumnado sea capaz de comprender y responder críticamente a los mensajes científicos de los medios de comunicación de manera activa (Jiménez-Liso et al., 2010, Reiss et al., 1999). En la literatura se ha destacado de manera extensa el interés didáctico de las noticias científicas en la enseñanza de la ciencia (McClune y Jarman, 2010; Oliveras et al., 2013), por lo que cabe esperar que este recurso sea también efectivo para el caso de la enseñanza de la NdC (García-Carmona, 2014). Sin embargo, las noticias no han sido redactadas para su incorporación en el currículo de ciencias (Jarman y McClune, 2007), por lo que también es necesario realizar un análisis para estudiar su potencial didáctico. 
Existen diversos trabajos que han empleado la NdC como estrategia para ejercitar activamente aspectos de interés. Así por ejemplo, Fernández-González (2009) empleó la posibilidad de vida extraterrestre para plantear cuestiones epistemológicas centradas en la función que desempeñan las hipótesis y el contraste permanente con los hechos que surgen. Su estrategia sitúa al alumnado en el papel de científico y le hace vivir el desarrollo del conocimiento y el mecanismo de su evolución. En otro trabajo, Romero Ariza y Vázquez Alonso (2013) desarrollan una propuesta didáctica con el fin de mejorar la comprensión de la NdC y ayudar al alumnado a aprender ideas adecuadas relacionadas con los conceptos de hipótesis, teoría y ley científica. Su metodología consiste en involucrar a los estudiantes en un proceso de investigación sobre la capacidad reproductiva de los dragones en el que tendrán que formular hipótesis, analizar datos y extraer conclusiones, de tal manera que se favorece el desarrollo de competencias científicas.

En base a todo lo anterior se planteó realizar este estudio sobre el potencial didáctico de las noticias de carácter científico-tecnológico de la prensa digital.

\section{Objetivos}

El objetivo general de este trabajo es analizar el potencial didáctico que poseen algunas noticias científicas de la prensa digital, como herramienta para introducir la NdC en la educación científica. Los aspectos que se pretenden abordar son los siguientes:

1. Identificar qué elementos de $\mathrm{NdC}$ están presentes en los artículos de las secciones de ciencia y tecnología de los diarios digitales de mayor difusión a nivel nacional.

2. Destacar su potencial aplicación para la introducción de la NdC en las aulas de ciencias.

\section{Naturaleza de la Ciencia}

La NdC integra características muy diversas y multidisciplinares, por lo que es necesario definir un marco teórico que fundamente y oriente el posterior análisis.

Aunque existen autores que identifican de manera restrictiva la NdC con la epistemología, es decir, el conocimiento en sí de la ciencia, en este trabajo se considera la visión ampliada del concepto, en la que se engloba, además, la sociología de la ciencia (Vázquez et al., 2007). La figura 1 muestra los componentes que conforman la NdC.

La epistemología de la ciencia abarca tres aspectos fundamentales: la naturaleza del conocimiento científico (NCC), los métodos de la ciencia (MC) y la evolución de la ciencia (EV).

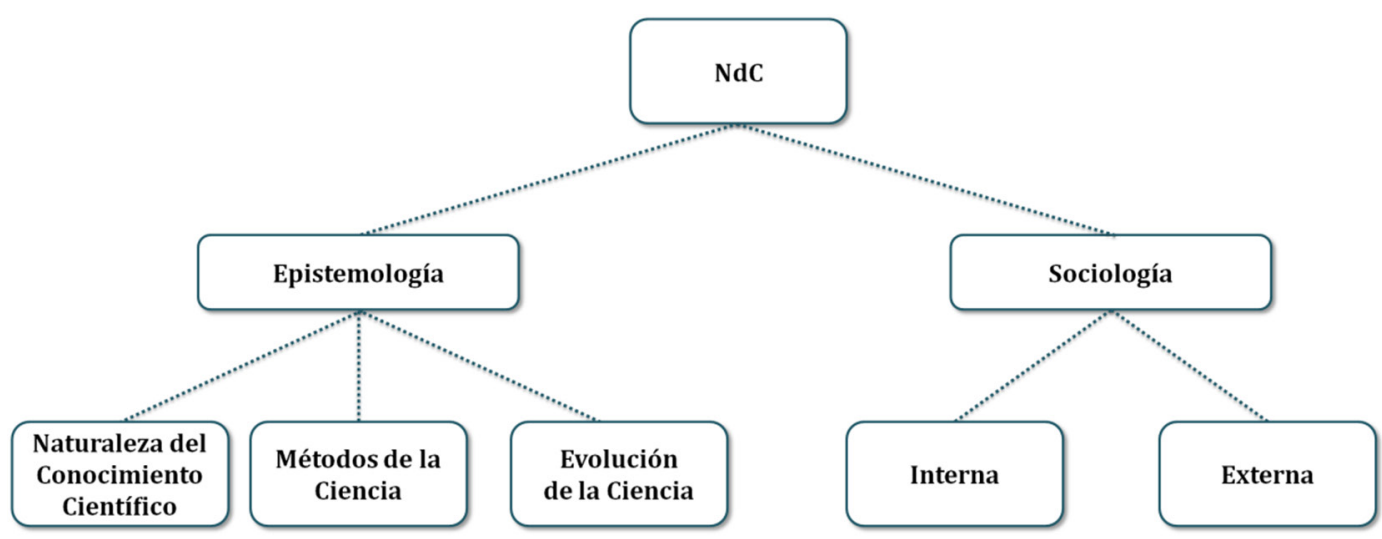

Figura 1. Diversos aspectos que engloba la $\mathrm{NdC}$ (Fuente: elaboración propia) 
La naturaleza del conocimiento científico se centra en temáticas relacionadas con las características y finalidad de este tipo de conocimiento. En este sentido, se acepta que el conocimiento científico debe cumplir dos condiciones fundamentales: ha de ser contrastable con la realidad (se ha de poder probar que en la realidad ocurre lo que se dice) y ha de ser reproducible (la contrastación se ha de poder realizar las veces que se desee, obteniendo siempre los mismos resultados).

En cuanto al método científico, lo primero es admitir que la ciencia no evoluciona siempre del mismo modo, por lo que se debería hablar de "los métodos" o "la metodología" de la ciencia. No obstante, por simplicidad, se habla del método científico. Lejos de tratarse de un método inductivo, como muchas veces se piensa pues así se enseña, la ciencia avanza por un método hipotético-deductivo (figura 2).

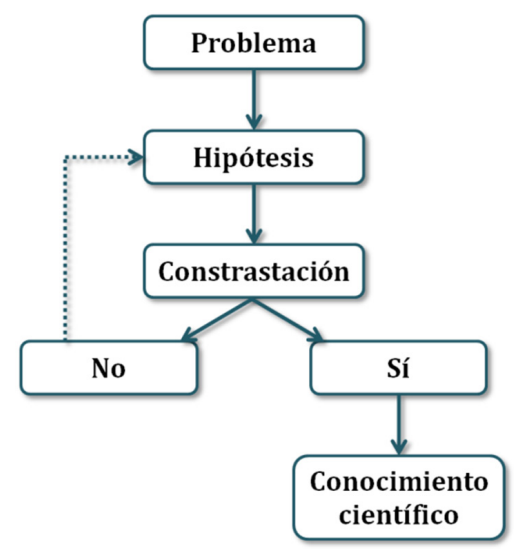

Figura 2. Esquema simplificado del método hipotético-deductivo (Fuente: elaboración propia)

El conocimiento científico surge de la necesidad de dar explicación a un problema, o un hecho concreto, que no la tiene con el conocimiento del momento. Para ello, se lleva a cabo la emisión de una hipótesis (conjetura) como posible respuesta, seguida de su contrastación con la realidad. Si la hipótesis queda refutada se habrá de plantear otra, y si queda confirmada se podrá considerar conocimiento científico una vez sea aprobado por la comunidad científica, cuestión que puede no ser inmediata. Cuando la hipótesis no se puede contrastar directamente, se realiza un proceso de deducción de consecuencias y son éstas las que se contrastan posteriormente. Por esta razón, el método se define como "deductivo", además de hipotético.

Por último, también forma parte de la epistemología todo lo relacionado con la evolución de la ciencia. A este respecto se puede decir que "la ciencia está en continua evolución" y que no existe un modo único para ello.

Finalmente, la sociología de la ciencia puede hacer referencia a su componente interna (las relaciones internas de la comunidad científica) o a la externa (en un sentido amplio, las relaciones entre ciencia, tecnología y sociedad - relaciones CTS -).

Tras definir los diferentes componentes de la NdC, y en base a la literatura (McComas y Olson, 1998; Bell, 2009; García-Carmona, 2014; García-Carmona, 2015), se pueden establecer además diferentes rasgos a considerar, como pueden ser el papel de la observación y experimentación, el carácter de las leyes y teorías científicas, el uso de modelos o la creatividad.

Este trabajo se centrará en el análisis de la presencia de cada uno de estos componentes y rasgos en artículos de prensa digital. 


\section{Metodología}

Se ha llevado a cabo un análisis de noticias de la prensa digital, con el fin de identificar elementos de la NdC para después emplearlos en el diseño de actividades. La investigación es de corte cualitativo, utilizando la técnica de análisis documental (Andréu, 2001; Rodríguez, 2007) con categorías inductivas extraídas de un protocolo que se detalla en los siguientes apartados. Se describe a continuación la elección de los diarios a analizar, la elección de la muestra y el procedimiento específico que se llevó a cabo para realizar el análisis.

\section{Elección de diarios}

Este trabajo se centra en la NdC en artículos de prensa digital. Para la elección de los diarios se llevó a cabo una revisión online para identificar cuáles presentan mayor influencia y visibilidad.

Un estudio realizado por Ramírez (2017), en el que se utilizaron varios indicadores clave o "KPI" de un total de 152 medios digitales, determinó los 10 periódicos digitales más influyentes. Por otro lado, según ComScore (herramienta de medición internacional de audiencias digitales homologada en España) los tres diarios digitales más leídos en diciembre de 2018 fueron abc.es (22 millones de usuarios), elpais.com (20,7 millones) y elmundo.es (20 millones) (vocento.com, 2019), que coinciden con los tres primeros del listado de Ramírez.

Se decidió, pues, que los diarios digitales analizados en este trabajo serían estos tres. Por lo tanto, nos centramos en el análisis de artículos relacionados con ciencia publicados en elpais.com, elmundo.es y abc.es.

\section{Muestra y procedimiento}

Se han analizado los artículos de la sección de ciencia de cada uno de los medios digitales seleccionados. En concreto, elpais.com presenta una sección titulada "ciencia", al igual que abc.es, mientras que elmundo.es presenta una sección titulada "ciencia y salud".

El estudio se realizó entre el 1 y el 7 de Abril de 2019. Los artículos se almacenaron como archivos .pdf y se clasificaron por días y por revista. Además, el análisis de contenido se ha practicado únicamente sobre contenido escrito, dejando fuera de este estudio los contenidos de figuras, audiovisuales e híbridos.

Se registraron un total de 53 noticias que incluían algún elemento relacionado con la $\mathrm{NdC}$. En concreto, 31 del abc.es, seis de elmundo.es y 16 de elpais.com. En el Anexo I se puede consultar el listado de las noticias analizadas Como instrumento de recogida de información se diseñó una ficha de análisis, que se presenta más adelante (tabla 1).

Para realizar el análisis se utilizó, a modo de protocolo, un documento inédito elaborado por el Prof. Dr. D. Manuel Fernández González, del Departamento de Didáctica de las Ciencias Experimentales de la Universidad de Granada (Anexo II).

El análisis se centró en determinar qué presencia tienen los aspectos de la NdC en la muestra de noticias seleccionada, atendiendo a la frecuencia de aparición, así como a su cantidad y variedad en una misma noticia. No se trató de encontrar qué aspectos son acordes con la visión actual de la NdC y cuáles podrían transmitir imágenes deformadas sobre la misma, sino de estimar qué aspectos de la NdC presenta el contenido de las noticias con el fin de utilizarlas en el aula como recurso para la enseñanza-aprendizaje de la NdC.

En el anexo III se muestra un ejemplo específico de cómo se ha aplicado este protocolo a una noticia concreta: Cientificos aseguran haber encontrado indicios de vida en Marte. En 
este anexo se exponen algunos fragmentos de la noticia relacionados con distintos rasgos de la NdC.

\section{Discusión de los resultados}

En primer lugar, se determinó la frecuencia de aparición de los distintos aspectos de la $\mathrm{NdC}$ en cada una de las revistas (tabla 1 ).

Tabla 1. Frecuencia con la que se alude a aspectos de la $\mathrm{NdC}(\mathrm{N}=53) . \mathrm{T} \rightarrow \mathrm{S}$, indica la influencia de la tecnología en la sociedad; $\mathrm{S} \rightarrow \mathrm{C}$, la de la sociedad en la ciencia; $\mathrm{S} \rightarrow \mathrm{T}$, la de la sociedad en la tecnología; y $C \rightarrow S$, la de la ciencia en la sociedad. (Fuente: Elaboración propia)

\begin{tabular}{|c|c|c|c|c|c|c|c|c|c|c|c|c|c|c|c|c|c|c|}
\hline \multirow[b]{3}{*}{ Revista } & \multicolumn{11}{|c|}{ Epistemología } & \multicolumn{7}{|c|}{ Sociología } \\
\hline & \multicolumn{4}{|c|}{ NCC } & \multicolumn{6}{|c|}{ MC } & \multirow[t]{2}{*}{ EV } & \multicolumn{4}{|c|}{ Externa } & \multicolumn{3}{|c|}{ Interna } \\
\hline & 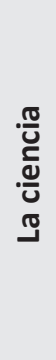 & 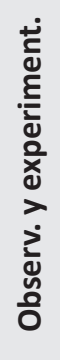 & 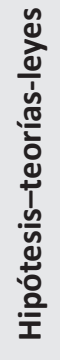 & 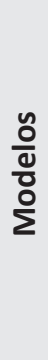 & 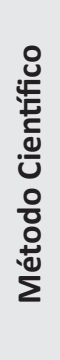 & 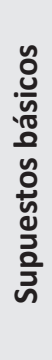 & 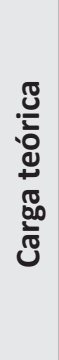 & 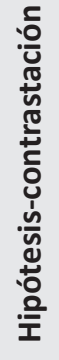 & 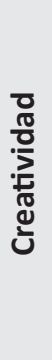 & 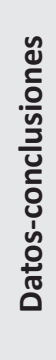 & & $\stackrel{n}{\uparrow}$ & $\begin{array}{l}u \\
\text { 勇 }\end{array}$ & 卡 & $\tilde{u}$ & 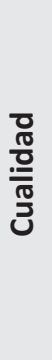 & $\begin{array}{l}\text { 莺 } \\
\frac{\text { on }}{0}\end{array}$ & \\
\hline$A B C$ & 15 & 4 & 24 & 2 & 5 & 52 & 0 & 22 & 0 & 33 & 3 & 3 & 4 & 0 & 5 & 1 & 9 & 16 \\
\hline EM & 0 & 0 & 1 & 0 & 0 & 3 & 0 & 7 & 0 & 5 & 1 & 0 & 1 & 0 & 1 & 0 & 2 & 0 \\
\hline EP & 14 & 5 & 9 & 0 & 1 & 17 & 0 & 12 & 0 & 7 & 4 & 3 & 15 & 0 & 8 & 6 & 7 & 1 \\
\hline Total & 29 & 9 & 34 & 2 & 6 & 72 & 0 & 41 & 0 & 45 & 8 & 6 & 20 & 0 & 14 & 7 & 18 & 17 \\
\hline $\begin{array}{l}\text { Por com- } \\
\text { ponente }\end{array}$ & \multicolumn{4}{|c|}{4} & \multicolumn{6}{|c|}{164} & 8 & \multicolumn{4}{|c|}{40} & \multicolumn{3}{|c|}{42} \\
\hline
\end{tabular}

\section{Epistemología vs sociología}

En la figura 3 se puede observar la comparación epistemología vs sociología de cada una de las tres revistas, y del conjunto.

Tal y como se puede observar, la epistemología está mucho más presente que la sociología en todos los casos, siendo el periódico elpais.es el que alcanza una mayor presencia de la sociología.

En la figura 4 se puede observar la comparación de la presencia de los diferentes componentes en cada una de las tres revistas, y del conjunto.

Para el abc.es, el componente más representado dentro de la epistemología es el método científico (56.6\%), y dentro de la sociología, su componente interna (13.1\%). Para elmundo.com, dentro de la epistemología el más presente es de nuevo el método científico (33.9\%), y dentro de la sociología ambos componentes están igualmente reflejadas. Para el país.es, el componente más reflejado de la epistemología es el método científico (33.9\%) aunque con presencia parecida a la NCC (25.7\%), y dentro de la sociología se trata más la sociología externa (23.9\%). 


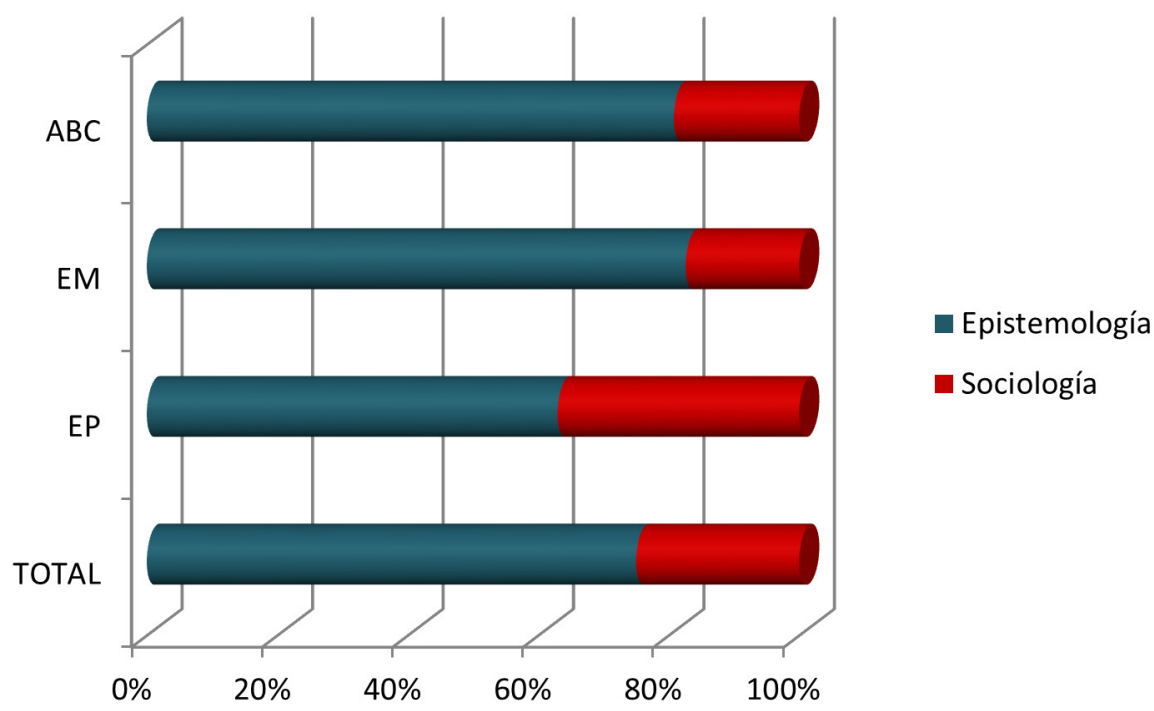

Figura 3. Comparación del análisis epistemología vs sociología (Fuente: elaboración propia)

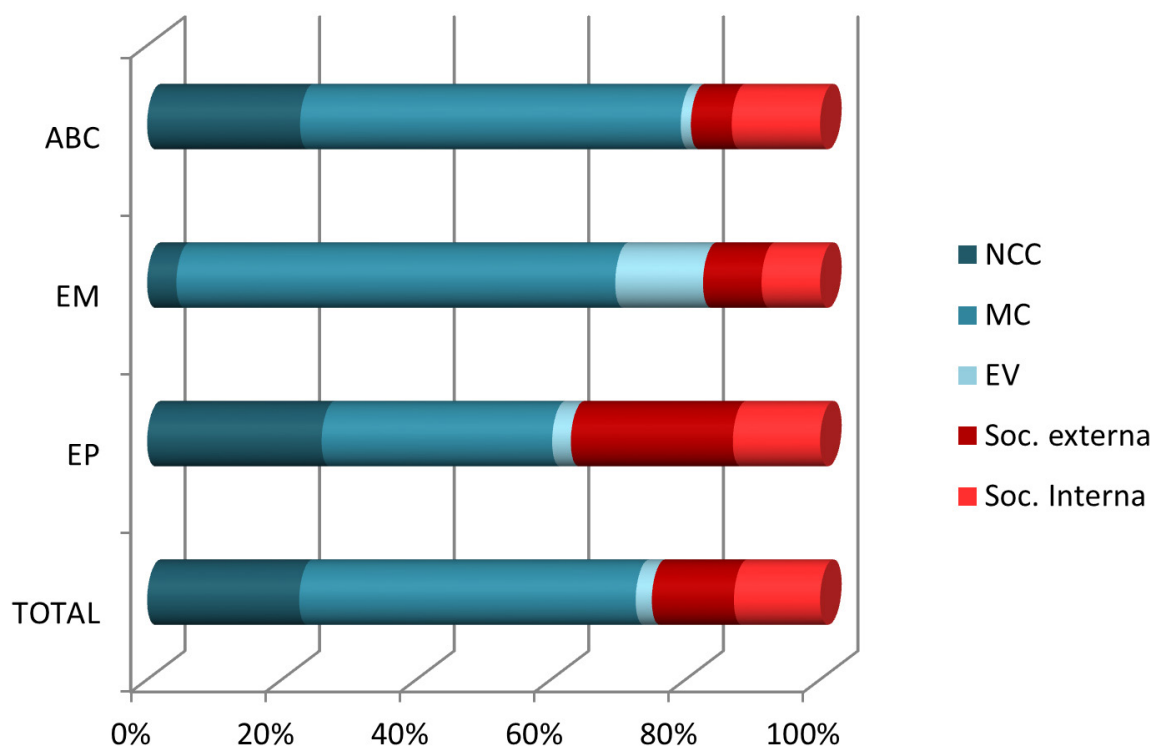

Figura 4. Comparación del análisis de los diferentes componentes de la NdC.

(Fuente: elaboración propia)

En cuanto a valores totales, dentro de la epistemología el elemento más reflejado es el método científico (50\%), y dentro de la sociología, la sociología interna (12.8\%), aunque prácticamente en igual medida que la externa (12.2\%).

En general, el MC es el componente más presente. Respecto a la sociología, en el análisis total y el mundo.com la sociología externa e interna tienen la misma presencia, mientras que para el abc.es tiene más presencia la interna, y para elpais.es, la externa con diferencia.

\section{Comparación de los diferentes elementos de la epistemología}

En la figura 5 se puede observar la comparación de la presencia de los diferentes elementos de la epistemología para cada una de las revistas, y para el conjunto. 


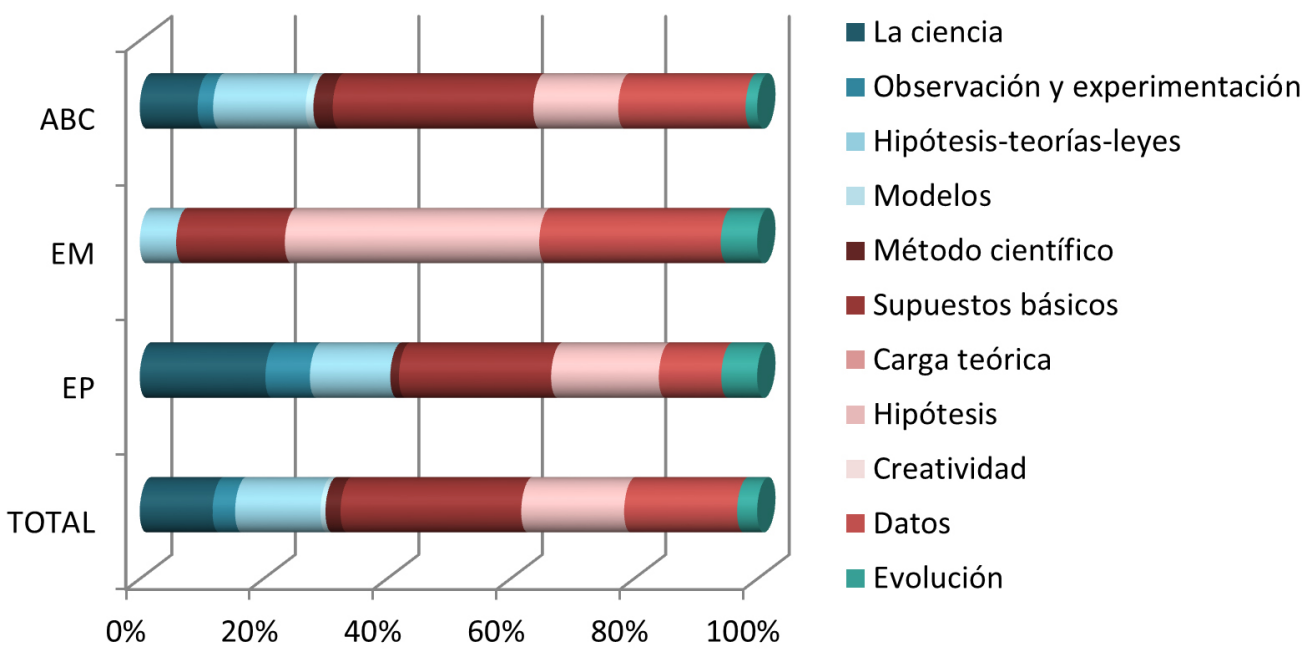

Figura 5. Comparación del análisis de los diferentes elementos de la epistemología.

(Fuente: elaboración propia)

Para el abc.es, el rasgo epistemológico más representado es el de los supuestos básicos, que forma parte del método científico e incluye el planteamiento del problema, los diseños experimentales y la realización de observaciones (Anexo II). Para elmundo.com, la hipótesis es la que aparece con más frecuencia (41.2\%). Finalmente, para elpais.es, el elemento más presente son los supuestos básicos (24.6\%), seguido de la ciencia (20.3 $\%)$ y la hipótesis (17.3\%). Con respecto al total, el elemento más tratado es, de nuevo, el relacionado con los supuestos básicos.

Así pues, los resultados muestran que el elemento más frecuente para el abc.es y elpais. es es el de supuestos básicos, mientras que el más aludido para elmundo.es es la hipótesis. El resto de componentes tienen una menor presencia, excepto los relacionados con la carga teórica y con la creatividad, que no tienen ninguna a pesar de ser esenciales para la génesis y evolución del conocimiento científico.

\section{Comparación de los diferentes elementos de la sociología}

La figura 6 muestra la comparación de los diferentes elementos de la sociología, para cada una de las revistas y para el conjunto.

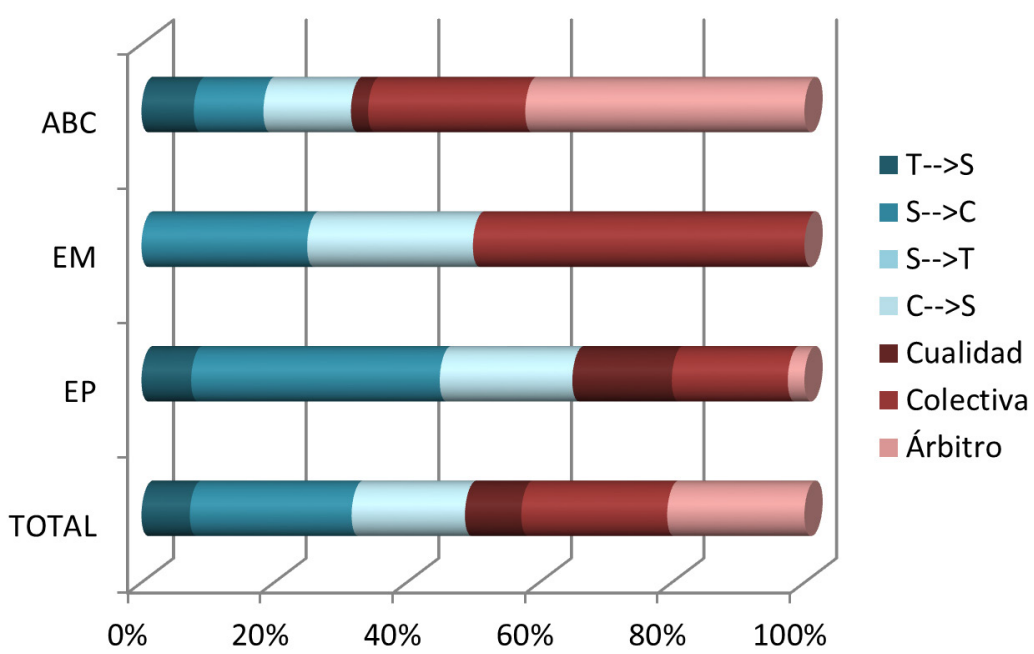

Figura 6. Comparación del análisis de los diferentes elementos de la sociología

(Fuente: elaboración propia) 
En el caso del abc.es, el elemento más frecuente es el definido como "árbitro", el cual está incluido dentro de la sociología interna y se relaciona con la compartición de nuevos conocimientos científicos y con el proceso necesario para que un nuevo conocimiento sea aceptado por la comunidad científica (Anexo II). Para elmundo.com, el más presente es el definido como "colectiva", que pertenece a la sociología interna y está relacionado con el carácter colectivo la ciencia. Para elpais.es, el más reflejado es el definido como $\mathrm{S} \rightarrow \mathrm{C}$, de la sociología externa, relacionado con la influencia de la sociedad sobre la ciencia. Finalmente, teniendo en cuenta el conjunto de noticias, el elemento más reflejado también es el $\mathrm{S} \rightarrow \mathrm{C}$.

\section{Conclusiones}

A partir de la necesidad de introducir la enseñanza de la $\mathrm{NdC}$ en el aula de ciencias, surge la propuesta de utilizar noticias científicas de la prensa digital. Este recurso es muy accesible y de fácil integración en los programas de enseñanza, y puede propiciar el análisis crítico-reflexivo en torno a qué es la ciencia, cómo avanza y qué factores influyen en ella. Sin embargo, las noticias no son redactadas pensando en su uso didáctico. Por tanto, el proceso de selección de las que podrían usarse requiere un análisis previo.

Del análisis realizado se pueden obtener varias conclusiones:

- La muestra de noticias seleccionadas ofrece información variada con alusiones a todos los componentes de la NdC y a prácticamente todos los elementos que han sido definidos en el marco teórico.

- Mediante el uso de un protocolo previamente definido, los diferentes aspectos de la $\mathrm{NdC}$ son identificables con relativa facilidad en la mayoría de las noticias, lo cual hace posible una reflexión en torno a los mismos.

- Las noticias contienen más de un aspecto de la $\mathrm{NdC}$ (en algunas se reconocen hasta ocho aspectos diferentes), lo que las hace adecuadas para enseñar sobre ellos.

- Aunque el repertorio del conjunto de noticias constituye una fuente de información bastante rica y completa, se podría llegar a la conclusión que elmundo.com no es la fuente más apropiada para su uso en el aula. Este hecho se debe a que tanto el número de noticias como la frecuencia de alusión a elementos de la $\mathrm{NdC}$ son muy reducidas.

En la tabla 2 se resumen aquellos aspectos que podrían presentar un potencial didáctico para cada una de las revistas y para el conjunto de ellas.

Tabla 2. Resumen de aspectos que podrían presentar potencial didáctico. (Fuente: elaboración propia)

\begin{tabular}{|c|c|c|}
\hline Revista & Epistemología & Sociología \\
\hline ABC & $\begin{array}{c}\text { Supuestos básicos } \\
\text { (planteamiento del problema, diseños } \\
\text { experimentales y realización de } \\
\text { observaciones) }\end{array}$ & $\begin{array}{c}\text { Árbitro } \\
\text { (compartición de nuevos conocimientos, } \\
\text { proceso de revisión) }\end{array}$ \\
\hline EM & Hipótesis & $\begin{array}{c}\text { Colectiva } \\
\text { (carácter colectivo de la ciencia) }\end{array}$ \\
\hline EP & Supuestos básicos & $\begin{array}{c}\mathrm{S} \rightarrow \mathrm{C} \\
\text { (influencia de la sociedad sobre la } \\
\text { ciencia) }\end{array}$ \\
\hline Total & Supuestos básicos & $\mathrm{S} \rightarrow \mathrm{C}$ \\
\hline
\end{tabular}


Por tanto, se puede concluir que el repertorio de noticias analizadas en este trabajo presenta buen potencial didáctico para integrar la NdC en el aula. Se espera por tanto que el profesorado se anime a emplearlo en sus clases ya que pueden ser utilizadas en la enseñanza de asignaturas relacionadas con disciplinas científicas.

\section{Limitaciones y futuras líneas de investigación}

Una limitación de este trabajo se relaciona con el hecho de que los documentos han sido analizados por un único codificador (previo consenso entre los autores firmantes en el análisis de varias noticias a modo de prueba piloto), lo que implica la no existencia de duplicidad en los procesos de análisis, aunque asegura el empleo del mismo criterio para todos los casos. Otra guarda relación con la muestra en sí y el periodo analizado. Sería necesario ampliar la muestra a un mayor número de diarios, así como una recogida de noticas durante un periodo de tiempo más largo. De este modo se procedería a un análisis más exhaustivo que permitiría disponer de una imagen más precisa de la NdC en las noticias de prensa.

Aunque no se ha profundizado en este aspecto, la información sobre la NdC que subyace en las noticias de la muestra es, en general, consonante con la visión actual de la NdC. Como futura línea de investigación también se puede plantear encontrar aquellos aspectos que son acordes con la visión actual de la NdC y cuáles podrían transmitir imágenes deformadas sobre la misma, para también usar posteriormente este análisis en el aula.

Por último, sería objeto de futuros trabajos el diseño de propuestas didácticas y el análisis de su efectividad a través de una validación de contenido por panel de expertos relacionados con la temática (es decir, profesorado de ciencias y de didáctica de las ciencias), seguida de una validación de constructo después de aplicar la propuesta en el aula.

\section{Agradecimientos}

Al grupo de Investigación HUM-613 (Didáctica de las Ciencias Experimentales y de la Sostenibilidad - Universidad de Granada), al proyecto RTI2018-094303-A-100 (Identificación de contextos científicos en la sociedad. Herramientas para docentes y ciudadanos - Ministerio de Ciencia, Innovación y Universidades) y al profesor Dr. D. Manuel Fernández González, del Departamento de Didáctica de las Ciencias Experimentales de la Universidad de Granada.

\section{Referencias bibliográficas}

Acevedo, J. A., Vázquez, A., Martín, M., Oliva, J. M., Acevedo, P., Paixao, M. F. y Manassero, M. A. (2005). Naturaleza de la ciencia y educación científica para la participación ciudadana. Una revisión crítica. Revista Eureka sobre Enseñanza y Divulgación de las Ciencias, 2(2), 121-140. Recuperado de: https://revistas.uca.es/index.php/eureka/ article/view/3912

Acevedo-Díaz, J. A. y García Carmona, A. (2016). Uso de la historia de la ciencia para comprender aspectos de la naturaleza de la ciencia. Fundamentación de una propuesta basada en la controversia Pasteur versus Liebig sobre la fermentación. CTS: Revista iberoamericana de ciencia, tecnología y sociedad, 11(33), 203-226. Recuperado de: $\mathrm{http}: / /$ hdl.handle.net/11441/67245

Acevedo-Díaz, J. A., García-Carmona, A. y del Mar Aragón, M. (2017). Historia de la ciencia para enseñar naturaleza de la ciencia: una estrategia para la formación inicial del profesorado de ciencia. Educación química, 28(3), 140-146. DOI: https://doi. org/10.1016/j.eq.2016.12.003 
Andréu, J. (2001). Las técnicas de análisis de contenido: Una revisión actualizada. Documento de trabajo, S2001/03, Centro de estudios andaluces. Recuperado de: https://campus.fundec.org.ar/admin/archivos/Andr\%C3\%A9u\%20Abela.\%20 Las\%20t\%C3\%A9cnicas\%20de\%20An\%C3\%A1lisis\%20de\%20Contenido.\%20 Una\%20revisi\%C3\%B3n\%20actualizada.pdf

Bell, R.L. (2009). Teaching the nature of science: Three critical questions. Best Practices in Science Education, 22, 1-6.

Campanario, J. M., Moya, A. y Otero, J. (2001). Invocaciones y usos inadecuados de la ciencia en publicidad. Enseñanza de las ciencias: revista de investigación y experiencias didácticas, 19(1), 45-56. Recuperado de: https://raco.cat/index.php/ Ensenanza/article/view/21709/21543

Driver, R. Leach, J. y Millar, R. (1996). Young people's images of science. McGraw-Hill Education (UK).

Fernández, I., Gil, D., Carrascosa, J., Cachapuz, A., y Praia, J. (2002). Visiones deformadas de la ciencia transmitidas por la enseñanza. Enseñanza de las Ciencias, 20(3), 477-488. Recuperado de: https://www.raco.cat/index.php/Ensenanza/article/ view/21841/21675

Fernández-González, M. (2009). ¿Vida extraterrestre? Aspectos epistemológicos para el aula. Revista Eureka sobre Enseñanza y Divulgación de las Ciencias, 6(3), 408-422. Recuperado de: http://hdl.handle.net/10498/9908

García-Carmona, A., Vázquez, A. y Manassero, M. A. (2011). Estado actual y perspectivas de la enseñanza de la naturaleza de la ciencia: una revisión de las creencias y obstáculos del profesorado. Enseñanza de las Ciencias, 28(3), 403-412. Recuperado de: https://www.raco.cat/index.php/Ensenanza/article/view/247899

García-Carmona, A. (2014). Naturaleza de la ciencia en noticias científicas de la prensa: análisis del contenido y potencialidades didácticas. Enseñanza de las Ciencias, 32(3), 493-509. Recuperado de: https://www.raco.cat/index.php/Ensenanza/ article/view/v32-n3-garcia-carmona

García Carmona, A. (2015). Noticias sobre temas de Astronomía en los diarios: un recurso para aprender sobre la naturaleza de la ciencia reflexivamente. Revista de Enseñanza de la Física, 27 (1), 19-30. Recuperado de: https://revistas.unc.edu.ar/index.php/ revistaEF/article/view/11408/11884

Jarman, R. y McClune, B. (2007). Do children really take note of science in the news? Primary Science Review, 98, 10-13.

Jiménez-Liso, M. R., Hernández Villalobos, L., y Lapetina, J. (2010). Dificultades y propuestas para utilizar las noticias científicas de la prensa en el aula de ciencias. Revista Eureka sobre Enseñanza y Divulgación de las Ciencias, 7(1), 107-126. Recuperado de: http://hdl.handle.net/10498/9866

Lederman, N.G. (2006). Research on nature of science: reflections on the past, anticipations of the future. Asia-Pacific Forum on Science Learning and Teaching, 7(1), 1-11.

Lederman, N. G. (2007). Nature of Science: Past, Present, and Future. Handbook of Research in Science Education, 2, 831-879.

Matthews, M.R. (1998). The Nature of Science and Science Teaching. En B.J. Fraser y K.G. Tobin (Eds.): International Handbook of Science Education, pp. 981-999. Kluwer Academic Publishers. 
McClune, B. y Jarman, R. (2010). Critical Reading of Science-Based News Reports: Establishing a knowledge, skills and attitudes framework. International Journal of Science Education, 32(6), 727-752. DOI: https://doi.org/10.1080/09500690902777402.

McComas W.F., Clough, M.P. y Almazroa, H. (1998). The Role and Character of The Nature of Science in Science Education. En W.F. McComas (Ed.): The Nature Of Science In Science Education. Rationales and Strategies, pp. 3-39. Dordrecht, The Netherlands: Kluwer Academic Publishers.

McComas, W.F. y Olson, J.K. (1998). The nature of science in international science education standards documents. En W.F. McComas (Ed.): The Nature Of Science In Science Education (pp. 41-52). Springer, Dordrecht.

Moreno, L. y Calvo, M. A. (2019). ¿Cómo presentan la historia de la química los libros de texto de Educación Secundaria? Un análisis desde la didáctica y los estudios históricos de la ciencia. Revista Eureka sobre Enseñanza y Divulgación de las Ciencias, 16(1),1101-1116 Recuperado de: https://revistas.uca.es/index.php/ eureka/article/view/4466/4127

Oliveras, B. Márquez, C. y Sanmartí, N. (2013). The use of newspaper articles as a tool to develop critical thinking in science classes. International Journal of Science Education, 35(6), 885-905. DOI: https://doi.org/10.1080/09500693.2011.586736

Ramírez, S. (2017). Sergioramirez.org: Los periódicos más influyentes en España en 2018. España. Recuperado de: https://sergioramirez.org/periodicos-digitales-espanoles

Reiss, M. J., Millar, R. y Osborne, J. (1999). Beyond 2000: Science/biology education for the future. Journal of biological education, 33(2), 68-70.

Rodríguez C., F.N. (2007). Generalidades acerca de las técnicas de investigación cuantitativa. Paradigmas, 2(1), 9-39.

Romero, M. y Vázquez, A. (2013). Investigando dragones: una propuesta para construir una visión adecuada de la Naturaleza de la Ciencia en Educación Secundaria. Revista Eureka sobre Enseñanza y Divulgación de las Ciencias, 10(1), 85-99. Recuperado de: https://revistas.uca.es/index.php/eureka/article/view/2796/2444

Spector, B., Strong, P. y La Porta, T. (1998). Teaching the nature of science as an element of science, technology and society. En W.F. McComas (Ed.): The Nature of Science in Science Education (pp. 267-276). Springer, Dordrecht. DOI: https://doi. org/10.1007/0-306-47215-5_17

Vázquez, A., Manassero, M. A, Acevedo, J. A. y Acevedo, P. (2007). Consensos sobre la Naturaleza de la Ciencia: la Comunidad Tecnocientífica. Revista Electrónica de Enseñanza de las Ciencias, 6(2), 331-363.

Vázquez, A. y Manassero, M. A. (2012). La selección de contenidos para enseñar naturaleza de la ciencia y tecnología (parte 1): Una revisión de las aportaciones de la investigación didáctica. Revista Eureka sobre Enseñanza y Divulgación de las Ciencias 9(1), 2-31. Recuperado de: http://hdl.handle.net/10498/14621

Vílchez González, J. M., Carrillo-Rosúa, J., Rodríguez Sabiote, C. y Jiménez-Tejada, M. P. (2015). Imagen de ciencia de estudiantes de Magisterio. Didáctica de las ciencias experimentales y sociales, 29, 157-172. DOI: https://doi.org/10.7203/DCES.29.4283

Vocento. (2019). Vocento.com: ABC.es Sites cerró 2018 aumentando su liderato digital en España. España. Recuperado de: https://www.vocento.com/abc-es-sites-cerro2018-aumentando-su-liderato-digital-en-espana/

Ziman, J. (2000). Real science: What it is and what it means. Cambridge University Press. 


\section{Anexos}

Anexo I. Listado de noticias analizadas

abc.es

1 de abril

- ¿'Perfeccionamos el arco y la rueda porque éramos muy listos o por imitación?

- El misterioso origen radiactivo de los rayos de las erupciones volcánicas.

- Hallan a las primeras víctimas del meteorito de los dinosaurios: murieron el mismo día.

- Los partidos políticos se comprometen a firmar el Pacto de Estado por la Ciencia que nunca llega.

- ¿Estamos a punto de ver la primera foto de un agujero negro de la Historia?

2 de abril

- ¿'Por qué no podemos evitar bailar cuando escuchamos música?

- Confirman la existencia del misterioso metano en Marte pero, ¿̇es una señal de vida?

- Se descubre una segunda galaxia sin materia oscura.

- Un pescador encuentra una cabeza de tiburón de 100 kg mordida por otro depredador más grande.

- Una científica calcula la temperatura interna de la luna.

3 de abril

- Desvelan el origen de la explosión más potente del Universo.

- El cromosoma que reveló los hijos secretos de Thomas Jefferson con una esclava.

- El telescopio Hubble explorará 200 binarios del Cinturón de Kuiper.

- Hallan los restos rituales de una misteriosa religión anterior a los Incas en el lago Titicaca.

- La NASA y el MIT muestran el futuro de la aviación: el avión con alas deformables.

4 de abril

- ¿Podrá la NASA poner hombres en Marte en 2033?

- Así podrían ser nuestras futuras casas en Marte.

- Resuelven uno de los mayores misterios del milenario ejército de terracota chino.

- Hawking se equivocaba: la materia oscura no está hecha de agujeros negros microscópicos.

- La Tierra «bola de nieve» se descongeló en un instante.

- Las dos galaxias sin materia oscura que desconciertan a los científicos.

- Observan nanopartículas formándose a nivel atómico y en tiempo real.

5 de abril

- ¿Quién era el misterioso hombre de Tongzi?

- Así podría ser el futuro de la Tierra: un planeta muerto junto al frío cadáver del Sol.

- Científicos aseguran haber encontrado indicios de vida en Marte.

- El infinito, un concepto de origen hindú que se puede observar en el cielo.

- Todo a punto para Cheops, la exploradora de exoplanetas liderada por España.

6 de abril

- Las terribles chinches gigantes que devoran serpientes, tortugas e incluso patos.

7 de abril

- La misteriosa ley matemática que parece regir el Sistema Solar.

- Siete mujeres matemáticas asombrosas que no deben ser olvidadas.

- Una máquina crea el genoma de un ser vivo artificial.

elmundo.es

1 de abril

- El primer genoma de un ser vivo diseñado al $100 \%$ por un ordenador.

- España tiene un gran desafío con su sistema energético. 
4 de abril

- El planeta que sobrevivió a la muerte de su estrella.

5 de abril

- Indonesia cerrará en 2020 la isla de Komodo a los turistas por el robo de dragones.

- La reina faraónica que recuperó su nombre y lugar en la historia cuatro milenios después.

7 de abril

- Muere Sydney Brenner, uno de los pioneros en biología molecular.

elpais.com

1 de abril

- Necesitamos menos toreros y más científicos en el Congreso.

- Así es 'Cheops', el satélite español para medir exoplanetas.

2 de abril

- La conciliación real no existe, ni para el hombre ni para la mujer.

- Las enigmáticas curaciones del cáncer.

- Los cristales plásticos, el futuro de la tecnología para enfriar las cosas.

- Por qué nos venden bolsas de patatas llenas de aire.

3 de abril

- ¿La luz de las pantallas daña la vista?

- La Gran Barrera australiana sufre una reducción de especies sin precedentes.

5 de abril

- Hallado el cadáver de un planeta como la Tierra.

- La promesa electoral sobre ciencia de todos los partidos es "imposible" de cumplir.

- Los tres 'funerales' que muestran la tristeza de los gorilas.

- Muere el Nobel Sydney Brenner, el científico que revolucionó la biología con un gusano.

6 de abril

- Es triste que haya tratamientos efectivos para el cáncer que muchos pacientes no pueden recibir.

- Hedwig Kohn, la investigadora judía que sobrevivió al régimen nazi.

- La aspirina brota de una cuenca minera asturiana.

7 de abril

- El sueño de una pastilla para ser feliz. 
Anexo II. Protocolo para el análisis de elementos de la NdC en documentos

Elaborado por D. Manuel Fernández González, del Departamento de Didáctica de las Ciencias Experimentales de la Universidad de Granada.

\section{EPISTEMOLOGÍA}

Naturaleza del conocimiento científico (NCC). Reflexión sobre la ciencia ya hecha.

1. La ciencia (el conocimiento científico, CC).

- Qué es y para qué sirve: Describe, explica y predice.

- Limitaciones de la ciencia: explica sólo parte de la realidad (Error: La ciencia y sus métodos pueden resolver todos los problemas)

- El CC tiene carácter tentativo y provisional. El CC está sujeto a cambios.

- Diferencias entre el conocimiento científico y otras formas de conocimiento (p.ej. pseudociencia)

- Ciencia-Tecnología: Interdependencia y diferencia entre ambas (Error: Ciencia y tecnología son lo mismo)

\section{Observación y experimentación.}

- El CC tiene base empírica (se observa y se experimenta).

- La ciencia se basa en la observación y la inferencia. Las inferencias son interpretaciones de las observaciones.

- El CC se basa fundamentalmente, pero no por completo, en observación, pruebas empíricas y argumentos racionales.

- Experimentos: el camino hacia el CC no siempre pasa por ellos (a veces la investigación científica utiliza sólo observaciones, otras, experimentos mentales).

- Error: Los experimentos son el principal camino hacia el conocimiento.

- Error: Toda investigación científica comienza por la observación sistemática del fenómeno estudiado.

\section{Hipótesis-teorías-leyes.}

- Qué es cada una. Relaciones mutuas. Las teorías contienen hipótesis y explican las leyes

- Las hipótesis/teorías explican, las leyes describen. Todas ellas predicen.

- Las leyes describen fenómenos. Las teorías los explican. Por tanto, las teorías no pueden convertirse en leyes. Son tipos de conocimiento diferentes. Las hipótesis, sin embargo, con pruebas que las apoyen, pueden convertirse en teorías (explicaciones) o leyes (descripciones).

- Las teorías explican sólo una parte de la realidad. Tienen carácter provisional.

- Las hipótesis (conjeturas), para ser científicas, han de ser susceptibles de comprobación. Tienen carácter tentativo y provisional.

- Las hipótesis/teorías han de estar en concordancia con los hechos. Tienen base empírica.

4. Modelos.

- Qué son. Explicación y predicción. Limitaciones (explican sólo una parte de la realidad).

- El modelo tiene base empírica // Si alguna hipótesis que sostiene al modelo falla, puede arrastrar la caída de éste // Puede haber más de un modelo interpretando el mismo fenómeno // El conocimiento es fruto de la interacción entre el pensamiento y la realidad.

- Relación modelo/teoría - realidad (Error: Las teorías científicas son un reflejo cierto de la realidad; Error: Los modelos representan la realidad).

Métodos de la Ciencia (MC). El proceso de hacer ciencia

\section{El método científico}

- No existe un único método con etapas bien definidas, sino una pluralidad de ellos.

- No existe una sola manera de hacer ciencia, por tanto, no hay ningún método científico universal en etapas sucesivas.

- Error: La eficacia del trabajo científico estriba en seguir fielmente las etapas ordenadas del método científico. 


\section{Supuestos básicos.}

- Planteamiento del problema (formular preguntas susceptibles de comprobación). Problema = hechos nuevos que no concuerdan con la teoría, o incoherencias de ésta

- Diseños experimentales (incluidos instrumentos). Estrategias de resolución: acotación de situaciones, determinación y control de variables, recogida y selección de datos. Experimentos mentales

- Realización de observaciones (p.ej. astronomía). Medición: precisión e incertidumbre.

\section{Carga teórica.}

- Subjetividad: la formulación de preguntas, la observación, la investigación, la selección e interpretación de datos y las conclusiones son guiadas por la teoría, o por ideas existentes

- Las perspectivas (teorías) y los científicos guían las observaciones e inferencias.

- Cuando se observa, cuando se seleccionan datos, se interpreta, pues la observación implica la construcción de significados.

- Error: El observador no debe actuar bajo la influencia de las teorías previas sobre el problema investigado.

4. Hipótesis (Modelos/Teorías) en acción.

- Planteamiento de hipótesis (Elaboración de modelos). Reformulación de hipótesis (si falla la primera).

- Inferencias (deducción de consecuencias de las hipótesis/modelos) y predicciones.

- Comprobación (contrastación) o refutación de las hipótesis. No siempre con experimentos, a veces sólo con observaciones

- Error: A través del experimento el investigador comprueba si la hipótesis es verdadera o falsa.

5. Creatividad. La investigación científica tiene carácter creativo.

6. Datos (una vez recogidos)/ Resultados.

- Análisis e interpretación de datos (ver MetC-3) $\rightarrow$ Resultados. A veces el examen de datos anteriores desde una nueva teoría contribuye al cambio en la ciencia.

- Interpretación de resultados = Conclusiones (derivadas de ellos, "basadas en pruebas"). El CC no surge directamente de los datos, sino tras un proceso de interpretación y construcción de teorías

- Los resultados no producen pruebas absolutas (pueden interpretarse de modo diferente). De los mismos datos es posible que los científicos den interpretaciones diferentes.

- Fiabilidad. Permitir la posibilidad de replicar los resultados repitiendo los experimentos. El resultado de un solo experimento pocas veces es suficiente para establecer un nuevo conocimiento.

- Error: la metodología científica garantiza totalmente la objetividad en el estudio de la realidad.

Evolución de la ciencia (EV), es decir, cómo la ciencia progresa.

- La ciencia revela un carácter evolutivo y revolucionario (McC.).

- Los debates entre teorías (o modelos) provocan el progreso de la ciencia

- Error: El conocimiento científico ha evolucionado históricamente por la acumulación de teorías verdaderas.

\section{SOCIOLOGÍA}

Sociología externa de la ciencia. Influencia de la sociedad sobre la ciencia/tecnología y viceversa

- $\quad \mathrm{T} \rightarrow \mathrm{S}$ : La tecnología forma parte de todos los aspectos de nuestras vidas. Proporciona a la sociedad los medios para mejorar o empeorar, según la manera de utilizarla.

- $\mathrm{S} \rightarrow \mathrm{T}$ : La sociedad vota a favor o en contra de ciertas tecnologías cada vez que compramos algo, es decir, basándose en lo que es importante para mejorar la vida.

- $S \rightarrow C$ : La ciencia está influida por la sociedad y la cultura donde se desarrolla. Los valores de la cultura determinan hacia dónde va la ciencia. 
- $\quad \mathrm{S} \rightarrow \mathrm{C}$ : La sociedad determina qué tipo de investigación es aceptable. Influye en la ciencia a través de las subvenciones.

- $\quad C \rightarrow S$ : La ciencia es parte de las tradiciones sociales y culturales. La ciencia influye en la sociedad porque capacita a las personas para poder conocer el mundo.

- $\quad C \rightarrow S$ : La ciencia da respuesta a las necesidades de las personas y mejora sus condiciones de vida.

- Interacción ciencia-tecnología-sociedad (CTS). La ciencia tiene implicaciones globales. La ciencia acomete los problemas globales a los que se enfrenta la humanidad (contaminación, cambio climático, recursos, residuos, etc.)

\section{Sociología interna de la ciencia}

1. Cualidades a señalar de los científicos (creatividad, ideología, valores...) (cualidad).

- El científico está condicionado por aspectos subjetivos y emocionales. A veces asume ideas impuestas por criterio de autoridad

- Los valores personales, las prioridades y las experiencias anteriores dictan hacia dónde y cómo los científicos dirigen su trabajo.

- Las decisiones en la aplicación del conocimiento científico y técnico no son neutrales, por tanto podrían entrar en conflicto con valores morales y éticos de diversos grupos sociales. Los científicos toman decisiones éticas.

- Error: Los científicos son especialmente objetivos.

2. Carácter colectivo de la construcción del conocimiento. La ciencia es pública, es decir, compartida (colectiva).

- El trabajo científico es una actividad colectiva y, a la vez, competitiva. Aunque algunos individuos pueden hacer contribuciones significativas, el trabajo científico se lleva a cabo con más frecuencia en grupo.

- Las personas de todas las culturas contribuyen a la ciencia.

3. La comunidad científica como árbitro de la aceptación de nuevos conocimientos (árbitro).

- Los nuevos conocimientos se comparten y deben superar un proceso de revisión crítica por los colegas para que sean aceptados por la comunidad científica.

- Error: La aceptación de nuevos conocimientos es inmediata. 
Anexo III. Tabla de ejemplo de aplicación del protocolo a una noticia concreta

\begin{tabular}{|c|c|}
\hline $\begin{array}{l}\text { Elementos de la } \\
\qquad \mathrm{NdC}\end{array}$ & Fragmentos de la noticia que los aluden \\
\hline NCC, la ciencia & $\begin{array}{c}\text { “¿Podemos estar ante la misma situación? ¿O puede que la nueva } \\
\text { metodología aportada por los húngaros revele algún misterio encerrado } \\
\text { en el meteorito ALH84001? Seguramente los científicos no tarden en } \\
\text { darnos una respuesta." }\end{array}$ \\
\hline NCC, hipótesis & $\begin{array}{l}\text { "El mundo más conocido a la Tierra del que tenemos pruebas no alberga } \\
\text { rastros orgánicos. O, por lo menos, no han sido hallados hasta ahora." }\end{array}$ \\
\hline $\begin{array}{l}\text { MC, supuestos } \\
\text { básicos }\end{array}$ & $\begin{array}{l}\text { "tras estudiar durante cuatro y seis años el suelo marciano en busca de } \\
\text { bacterias, los resultados fueron decepcionantes. El mundo más conocido } \\
\text { a la Tierra del que tenemos pruebas no alberga rastros orgánicos." }\end{array}$ \\
\hline MC, hipótesis & $\begin{array}{l}\text { "Su investigación también sugiere, además, que puede haber existido } \\
\text { vida en otros planetas." }\end{array}$ \\
\hline MC, conclusiones & $\begin{array}{l}\text { "algunos seres habrían habitado Marte en la antigüedad. Al menos } \\
\text { bacterias. Así lo afirman científicos de Hungría que aseguran haber } \\
\text { encontrado huellas de material orgánico incrustadas en forma } \\
\text { mineralizada en un meteorito marciano localizado a finales de los años } \\
70 . "\end{array}$ \\
\hline $\begin{array}{l}\text { Evolución de la } \\
\text { ciencia }\end{array}$ & $\begin{array}{c}\text { "Esta nueva investigación podría cambiar el examen de los meteoritos en } \\
\text { el futuro." }\end{array}$ \\
\hline $\begin{array}{l}\text { Sociología externa, } \\
\qquad \mathrm{C} \rightarrow \mathrm{S}\end{array}$ & $\begin{array}{c}\text { "La investigación también será de interés para los planetólogos, expertos } \\
\text { en meteoritos y astrobiología, así como para los investigadores del origen } \\
\text { de la vida, y para el público en general,..." }\end{array}$ \\
\hline $\begin{array}{l}\text { Sociología interna, } \\
\text { árbitro }\end{array}$ & $\begin{array}{c}\text { "Y, según un estudio publicado en marzo en la revista «Open } \\
\text { Astronomy»,..." }\end{array}$ \\
\hline $\begin{array}{l}\text { Sociología interna, } \\
\text { colectiva }\end{array}$ & $\begin{array}{c}\text { "El nuevo estudio, de los autores Ildiko Gyollai, Márta Polgári y Szaniszló } \\
\text { Bérczi,..." }\end{array}$ \\
\hline
\end{tabular}


Anexo IV. Tabla análisis de noticias. Cada número representa la frecuencia con la que determinado componente de la $\mathrm{NdC}$ aparece en esa noticia

\begin{tabular}{|c|c|c|c|c|c|c|c|c|c|c|c|c|c|c|c|c|c|c|}
\hline \multirow[b]{3}{*}{ Artículo } & \multicolumn{11}{|c|}{ Epistemología } & \multicolumn{7}{|c|}{ Sociología } \\
\hline & \multicolumn{4}{|c|}{ NCC } & \multicolumn{6}{|c|}{ MC } & \multirow[t]{2}{*}{ EV } & \multicolumn{4}{|c|}{ Externa } & \multicolumn{3}{|c|}{ Interna } \\
\hline & $\begin{array}{l}\bar{a} \\
\frac{2}{\bar{D}} \\
\frac{2}{3} \\
\frac{2}{0} .\end{array}$ & 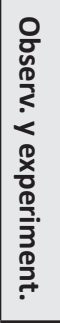 & 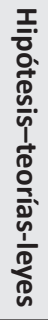 & $\begin{array}{l}\frac{3}{\circ} \\
\frac{0}{0} \\
\frac{0}{0}\end{array}$ & กิ & 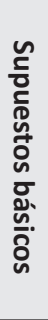 & 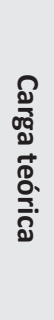 & 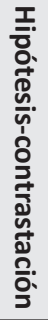 & 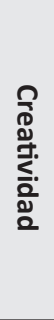 & 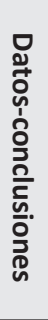 & & $\begin{array}{l}\dashv \\
\downarrow \\
\sim\end{array}$ & $\begin{array}{l}n \\
\downarrow \\
n\end{array}$ & $\begin{array}{l}\sim \\
\downarrow \\
-1\end{array}$ & $\begin{array}{l}n \\
\downarrow \\
\sim\end{array}$ & 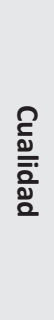 & 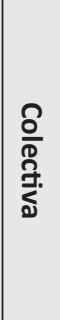 & 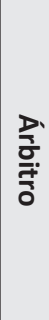 \\
\hline ABC_010419_1 & & 1 & & & 1 & 2 & & 2 & & 2 & & & & & & & & \\
\hline ABC_010419_2 & & & 2 & & & 2 & & 1 & & 2 & & & & & & & & \\
\hline ABC_010419_3 & 1 & & & & & & & & & & & & & & & 1 & 1 & 1 \\
\hline ABC_010419_4 & & & & & & & & & & & & & 1 & & & & & \\
\hline ABC_010419_5 & & & & 1 & & 1 & & & & & & & & & & & & \\
\hline ABC_020419_1 & & & & & 1 & 1 & & 2 & & 4 & & & & & 1 & & 1 & 2 \\
\hline$A B C 0204192$ & & 1 & 2 & & & 4 & & 3 & & 3 & & & & & & & 1 & 1 \\
\hline$A B C \quad 02041933$ & & & & & & & & & & 1 & & & & & & & & \\
\hline ABC_020419_4 & & & & & & & & 1 & & & & & & & & & & \\
\hline ABC_020419_5 & & & & & & 1 & & & & & & & & & & & & \\
\hline ABC_030419_1 & 2 & & 2 & 1 & 1 & 5 & & & & 2 & & & & & & & & 1 \\
\hline ABC_030419_2 & 2 & & & & & & & & & 2 & & & & & & & & 1 \\
\hline$A B C=0304193$ & & & & & & 2 & & & & & & & & & & & & \\
\hline ABC_030419_4 & 1 & & & & & 1 & & & & 1 & & & & & & & & 1 \\
\hline ABC_030419_5 & & & & & & & & & & & & 1 & & & & & & 1 \\
\hline ABC_040419_1 & & & & & & 1 & & & & & & & 2 & & 1 & & & \\
\hline$A B C+040419 \_2$ & & & & & & 1 & & 1 & & & & & & & & & 1 & \\
\hline ABC_040419_3 & 2 & & 3 & & 1 & 2 & 1 & & & 1 & & & & & & & & 1 \\
\hline ABC_040419_4 & 1 & & 3 & & & 2 & & 2 & & 1 & & & & & & & 1 & 1 \\
\hline ABC_040419_5 & 1 & & 2 & & & 3 & & & & 1 & & & & & 1 & & & \\
\hline ABC_040419_6 & & 1 & & & & & & & & 1 & & & & & & & & 1 \\
\hline ABC_040419_7 & & & & & & & & & & & & & & & 1 & & & \\
\hline ABC_050419_1 & & & 1 & & 1 & 2 & & 4 & & 3 & & & & & & & 1 & 1 \\
\hline$A B C 0504192$ & & & 3 & & & 7 & & & & 1 & & & & & & & 1 & 1 \\
\hline ABC_050419_3 & 1 & & 1 & & & 4 & & 4 & & 5 & 1 & & & & 1 & & 2 & 1 \\
\hline ABC_050419_4 & 1 & 1 & 1 & & & 2 & & & & 1 & & & & & & & & \\
\hline$A B C 050419 \_5$ & 2 & & 1 & & & 4 & & 1 & & & 1 & & & & & & & \\
\hline ABC_060419 & & & & & & 1 & & & & 1 & & & & & & & & 1 \\
\hline ABC_070419_1 & & & 2 & & & 2 & & 1 & & & & & & & & & & \\
\hline$A B C=070419 \_2$ & & & & & & & & & & & & & 1 & & & & & \\
\hline ABC_070419_3 & 1 & & 1 & & & 2 & & & & 1 & 1 & 2 & & & 2 & & & 1 \\
\hline EM_010419_1 & & & & & & 1 & & 1 & & 1 & & & & & 1 & & & \\
\hline EM_010419_2 & & & & & & & & & & & & & & & 1 & & & \\
\hline EM_040419 & & & 1 & & & 1 & & 4 & & 4 & 1 & & & & & & & \\
\hline EM_050419_1 & & & & & & & & 1 & & & & & 1 & & & & & \\
\hline EM_050419_2 & & & & & & 1 & & 1 & & & & & & & & & & \\
\hline EM_070419 & & & & & & & & & & & & & & & & & 2 & \\
\hline EP_010419_1 & & & & & & & & & & & & & 1 & & & & & \\
\hline EP_010419_2 & & & 2 & & & 2 & & 1 & & 2 & & & & & & & 2 & \\
\hline EP_020419_1 & & & 1 & & 1 & 1 & & & & & & & 6 & & & 3 & & \\
\hline EP_020419_2 & 5 & & 3 & & & & & & & & 1 & & & & 1 & & & \\
\hline EP_020419_3 & & & & & & 2 & & 2 & & & & 3 & & & & & & 1 \\
\hline EP_020419_4 & & & & & & 4 & & & & & & & 1 & & & & & \\
\hline EP_030419_1 & & 1 & & & & 1 & & 1 & & 1 & & & & & & & & \\
\hline EP_030419_2 & & 1 & & & & 3 & & 3 & & 1 & & & & & & & & \\
\hline EP_050419_1 & & 1 & 1 & & & 1 & & 3 & & 2 & & & & & & & & \\
\hline EP_050419_2 & & & & & & & & & & & & & 1 & & & & & \\
\hline EP_050419_3 & & 1 & & & & & & & & 1 & & & & & & & & \\
\hline EP_050419_4 & 6 & 1 & & & & 2 & & 2 & & & 1 & & 1 & & 1 & & 4 & \\
\hline EP_060419_1 & & & 2 & & & 1 & & & & & 1 & & 1 & & 3 & 2 & & \\
\hline EP_060419_2 & 1 & & & & & & & & & & 1 & & 4 & & & 1 & & \\
\hline EP_060419_3 & 1 & & & & & & & & & & & & & & 1 & & 1 & \\
\hline EP_070419 & 1 & & & & & & & & & & & & & & 2 & & & \\
\hline
\end{tabular}


